\title{
Characterization of He Bubbles in Irradiated Aluminium with High Resolution Transmission Electron Microscopy and Electron Energy Loss Spectroscopy
}

\author{
David Rondón Brito ${ }^{1}$, Esteban A. Sánchez ${ }^{2}$ and Alfredo Tolley ${ }^{2 *}$ \\ 1. Institutlo Balseiro, Comisión Nacional de Energía Atómica (CNEA) and Universidad Nacional de \\ Cuyo, San Carlos de Bariloche, Argentina (presently at Centro Atómico Ezeiza, CNEA, Ezeiza, \\ Argentina). \\ 2. Centro Atómico Bariloche CNEA-CONICET, San Carlos de Bariloche, Argentina. (12pt) \\ * Corresponding author: tolley@cab.cnea.gov.ar
}

Irradiation effects in metallic alloys has been an active area of research since a few decades ago, and is actually stimulated by the demand of developing materials that should withstand mechanical integrity at higher temperatures and higher irradiation doses for structural components of Generation IV fission reactors and for first wall components in fusion reactors [1].

This work reports results of $20 \mathrm{keV} \mathrm{He}{ }^{+}$irradiations on high purity aluminium at room temperature. The aim of the experiments was to study the formation of nanometer sized bubbles that cause swelling and embrittlement, and the effects of blistering that results from coalescence of bubbles and leads to surface erosion by exfoliation. The irradiation experiments were carried out in a $120 \mathrm{kV}$ ion accelerator at Centro Atómico Bariloche. A Tecnai F20 transmission electron microscope equipped with a Quantum ER system was used for post-irradiation characterization, combining High Resolution Imaging (HRTEM) and Electron Energy Loss Spectroscopy (EELS).

Figure 1a shows faceted He bubbles in the aluminium matrix, imaged with HRTEM, produced by an irradiation fluence of $5.2 \times 10^{16}$ ions $/ \mathrm{cm}^{2}$, equivalent to an irradiation dose of 3 displacements per atom (dpa). Figure $1 \mathrm{~b}$ corresponds to a specimen irradiated to a fluence of $2.6 \times 10^{17} \mathrm{ions} / \mathrm{cm}^{2}$ that corresponds to $15 \mathrm{dpa}$, where faceting is more marked. The mean equivalent diameter at $15 \mathrm{dpa}$ (that of a spherical bubble with the same volume as the faceted bubble) was $(2.4 \pm 0.1) \mathrm{nm}$, and the width of the size distribution was $0.6 \mathrm{~nm}$ (figure 1c).

Figure 1d shows three EELS spectra for comparison obtained in i) an unirradiated pure Al specimen; ii) an irradiated $\mathrm{Al}$ specimen to a dose of $3 \mathrm{dpa}$ and iii) an irradiated Al specimen to a dose of $15 \mathrm{dpa}$. In all spectra, typical Al volume plasmon peaks at energy losses of about $15 \mathrm{eV}$ and $30 \mathrm{eV}$ are clearly observed. In between these plasmon peaks, in the irradiated Al specimens a small peak can be observed. The inset in figure 1d shows a detail of the energy loss region between the plasmon peaks for the three specimens mentioned. The curves have been displaced for clarity. The small peak in the irradiated specimens corresponds to the excitation of the $\mathrm{K}$ shell of He. The energy of the small $\mathrm{K}$ shell He peak was found to vary between $21.5 \mathrm{eV}$ and $24 \mathrm{eV}$. Such a variation in the energy of the K shell He peak in He bubbles formed by ion implantation has been previously reported by Jaegger and co-workers [2], and was attributed to differences in the gas pressure inside the bubbles.

Summarizing the present results, He in irradiated Al has been detected by means of EELS. Further studies involving quantification of the He content as a function of the irradiation parameters are in progress. 


\section{References:}

[1] S.J.Zinkle, G.S.Was, Acta Materialia 61, (2013) p. 735.

[2] W.Jaegger et al., J. Nuc. Mater. 111\&112, (1982) p. 674.

[3] The authors acknowledge financial support from CNEA and the ANPCyT through grant PICT(2015) 1641.

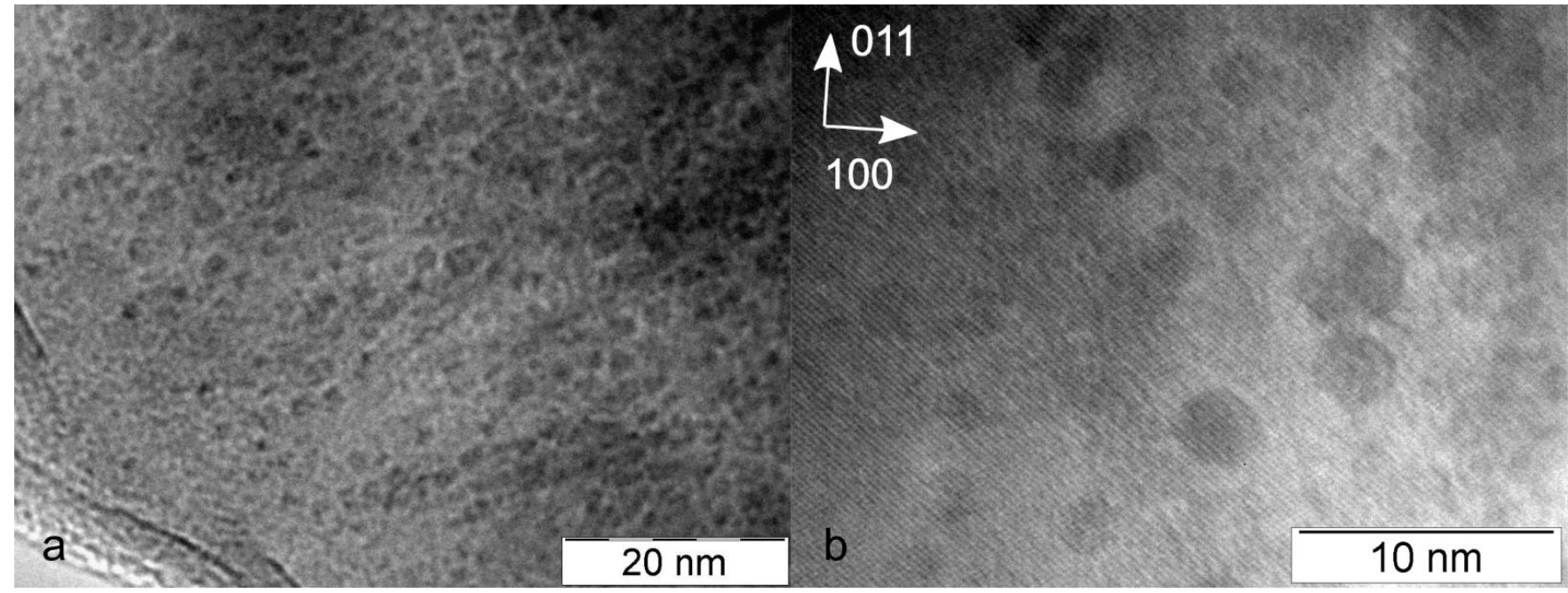

C
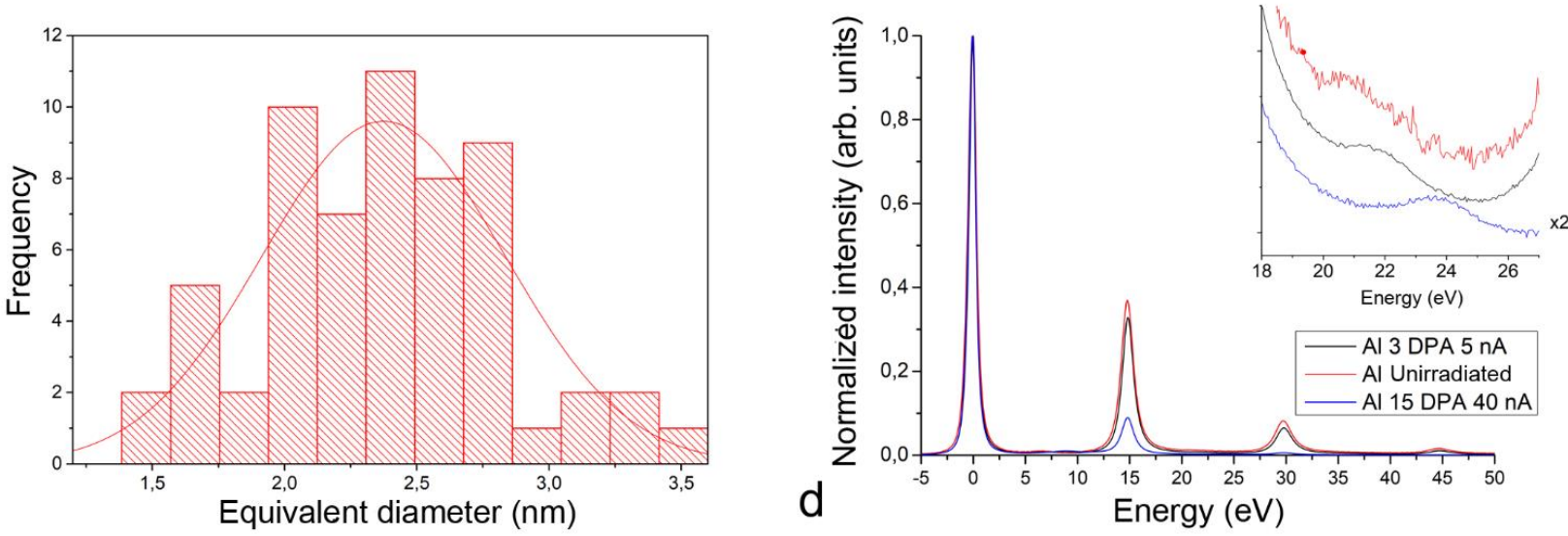

Figure 1: a) He bubbles in high purity $\mathrm{Al}$ irradiated with $20 \mathrm{keV}$ He ions to a dose of $3 \mathrm{dpa}$. The bubbles are equiaxed and show a tendency to form facets. b) He bubbles in high purity $\mathrm{Al}$ irradiated to a dose of 15 dpa. The bubbles are larger in size and show clear facets. c) Size distribution of the He bubbles corresponding to image $b$ ). The diameter indicated corresponds to the equivalent diameter of a spherical bubble with the same volume as the faceted bubble. d) EELS spectra of irradiated high purity Al to doses of $3 \mathrm{dpa}$ and $15 \mathrm{dpa}$. A spectra of an unirradiated $\mathrm{Al}$ specimen is shown for comparison. The inset shows the energy loss region between $18 \mathrm{eV}$ and $26 \mathrm{eV}$ in detail. 